\title{
Vibrational modes of light nuclei
}

\author{
Lorenzo Fortunato ${ }^{1,2, *}$ \\ ${ }^{1}$ Dipartimento di Fisica e Astronomia, "G. Galilei", University of Padova, (Italy) \\ ${ }^{2}$ I.N.F.N. -Sez. di Padova, via Marzolo, 8, I-35131, Padova (Italy)
}

\begin{abstract}
A potential energy surface is constructed for various light nuclei, ${ }^{3,4} \mathrm{He}$ with the Argonne V18 potential and then for alphaconjugate nuclei like ${ }^{12} \mathrm{C},{ }^{16} \mathrm{O},{ }^{20} \mathrm{Ne}$ with $\alpha-\alpha$ potentials. Despite the nucleons have large momenta and large fluctuations around the equilibrium points, the minimization suggests the underlying geometric structure. We calculate the Hessian matrix determining the normal modes of vibration. This approach might lead to a new way of fitting the alpha-alpha interactions on the spectroscopic data available for alpha-conjugate nuclei, rather than on scattering phase shift.
\end{abstract}

\section{Introduction}

Molecular models of nuclei are fundamentally different from models used in molecular physics, where the Born-Oppenheimer approximation is valid and one can think of nuclear motion as a small vibration, happening only close to the minimum of a very deep potential (in molecular energy scales)[1]. Nuclei have large kinetic energy $\langle T\rangle$, comparable to the potential energy $\langle V\rangle$ and the zero point motion inside the Potential Energy Surface (PES, for short) is a large fraction of the well depth, therefore there are large fluctuations around the equilibrium points and we should not expect that the vibrational levels are deeply lying in the potential well as it happens in molecules. At most they can be weakly bound states, close to threshold, or more probably resonances in the continuum. Despite this fact, it is instructive to look at the normal modes, find the "best" internal coordinates to express symmetry-adapted vibrational motio and calculate the energy scale and structure of the vibrational levels.

\section{Light nuclei: ${ }^{4} \mathrm{He}$ and ${ }^{3} \mathrm{He}$}

I take Argonne V18 nucleon-nucleon interaction as a well-established potential among all the constituents of the $\alpha$ particle, although in principle all the results are model dependent and the addition of a three body potential still hasn't been investigated in this context. For the moment let's consider only S-wave potentials. It's very interesting to consider also P-waves and other terms, they might lead to conical interesection (see quantum chemistry). With the above interactions, we build up a

*e-mail: fortunat@pd.infn.it 
global PES:

$$
\operatorname{PES}\left(\vec{r}_{1}, \vec{r}_{2}, \vec{r}_{3}, \vec{r}_{4}\right)=\sum_{i<j}^{4} V_{i j}\left(\left|\vec{r}_{i}-\vec{r}_{j}\right|\right) \quad \sum^{4} m_{i} \vec{r}_{i}=0
$$

that must be minimized with the constraints imposed by the center of mass. Brute force minimization for the ${ }^{4} \mathrm{He}$ case gives a minimum at $-557.531 \mathrm{MeV}$ and a set of equilibrium coordinates. These coordinates are not unique, there are still a number of rotations that give equivalent sets (loci), therefore one can impose certain other conditions, while keeping the minimum at the same energy. The following list of constraints can be imposed on minimization without lifting the minimum:

$$
x_{1}=-x_{2} \quad y_{1}=-y_{2} \quad z_{1}=z_{2} \quad x_{3}=-x_{4} \quad y_{3}=-y_{4} \quad z_{3}=z_{4}
$$

One easily gets the positions of the equilibrium points, around which the nucleons move dynamically with (large) fluctuations.

$$
\begin{array}{ccc}
x_{1}=-0.437648 \mathrm{fm} & y_{1}=0 \mathrm{fm} & z_{1}=-0.31883 \mathrm{fm} \\
x_{3}=0.0446687 \mathrm{fm} & y_{3}=-0.43476 \mathrm{fm} & z_{3}=0.318391 \mathrm{fm}
\end{array}
$$

They describe a shape that is close to a tetrahedron. There are two differences: the distance between the protons' equilibrium points is larger than the corresponding distance between the neutrons and the differences in spin-spin channels are such that the nn edge is rotated $5.87^{\circ}$ away from being orthogonal to the pp edge. See Fig. 1.

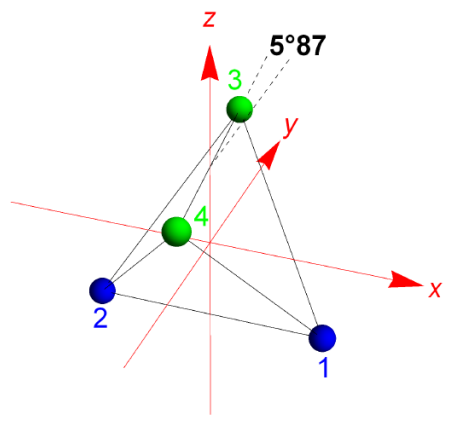

Figure 1. Equilibrium shape of ${ }^{4} \mathrm{He}$. Protons' equilibrium points in blue.

A clooser look at these values reveals that they satisfy only the geometric requirements of the discrete point-group $C_{2}$ that contains only the operations identity (E) and $180^{\circ}$ rotation around the symmetry axis (C2). This group has only one even $(A)$ and one odd $(B)$ representations (the multiplication table is trivial).

The diagonalization of the mass-corrected Hessian matrix $\tilde{H}_{l k}=H_{l k} / \sqrt{m_{l} m_{k}}=$ $\left(\partial^{2} P E S / \partial q_{l} \partial q_{k}\right) / \sqrt{m_{l} m_{k}}$ gives the normal modes: it corresponds to pure harmonic vibrations (approximation of the full PES with quadratic functions around the minimum), clearly too drastic for nuclei because the energy levels are almost at threshold. A better approach is of course the full diagonalization of the 4-body problem with kinetic terms + potentials. In any case group theory (as in molecular physics) gives the characters of normal modes of vibrations. They turn out to be very high in energy: 
A $689.056 \mathrm{MeV}$ (this is the totally symmetric breathing mode), B $526.534 \mathrm{MeV}, \mathrm{A}$ 525.475 MeV, B 392.526 MeV, A $388.906 \mathrm{MeV}, \mathrm{A} 285.293 \mathrm{MeV}$, and therefore most problably not observable.

The same minimization with constraints on ${ }^{3} \mathrm{He}$, gives the minimum at -277.67 $\mathrm{MeV}$ with planar geometry, but not quite isosceles! Again the spin-spin interaction is slightly different and the coordinates of the equilibrium points are in this case:

$$
\begin{array}{ccc}
x_{1}=0.283102 \mathrm{fm} & y_{1}=0.436916 \mathrm{fm} & z_{1}=0 . \mathrm{fm} \\
x_{2}=0.232502 \mathrm{fm} & y_{2}=-0.436916 \mathrm{fm} & z_{2}=0 . \mathrm{fm} \\
x_{3}=-0.514895 \mathrm{fm} & y_{3}=0 . \mathrm{fm} & z_{3}=0 . \mathrm{fm}
\end{array}
$$

The point-group symmetry is just $C_{s}$, i.e. the group of identity and reflection upon the horizontal plane and the 3 normal modes are all of $A^{\prime}$ type with very high energies: 642.033, 1081.92 and $1856.07 \mathrm{MeV}$ (breathing). Once again the eigenenergies are clearly too high for nuclear dynamics (they occur in a regime that is hundreds of $\mathrm{MeV}$ above separation threshold), but in principle they should exist anyway.

\section{Alpha-conjugate nuclei}

In general by incresing the mass of the clusters, their vibrational energies become lower, so maybe these kind of molecular vibrations are observable for heavier nuclei. Bijker and Iachello[2] have clearly demonstrated the succesfull application of the ACM, or algebraic cluster model, to the vibrational-rotational spectrum of $\alpha-\alpha$ conjugate nuclei like ${ }^{12} \mathrm{C}$ and ${ }^{16} \mathrm{O}$. Therefore it looks promising to try to infer an analytic form for the $\alpha-\alpha$ potential that fits the two lowest vibrations in ${ }^{12} \mathrm{C}$ or the three lowest vibrations of ${ }^{16} \mathrm{O}$, or simoultaneously both of them and try to compare with other models derived from phase-shifts. A suggested form is similar to the Ali-Bodmer potential, $V_{1} e^{-a_{1} r^{2}}+V_{2} e^{-a_{2} r^{2}}+$ Coulomb repulsion between charged spheres. Surprisingly, among all possible potentials with two gaussians, ${ }^{12} \mathrm{C}$ is reproduced very well with a potential that is quite shallow and has no nuclear repulsion at short distances. We get the A-bandhead at $7.30(\mathrm{MeV})$ and the E-bandhead at 10.40 $\mathrm{MeV}$, to be compared with experimental values: 7.65 and $10.84 \mathrm{MeV}$.

Instead of looking at the Hessian matrix, one would ideally like to solve the Schroedinger equation for these systems (this has already been done for g.s. and lowest exc.st. in $\mathrm{A}=3,4$ with this potential) but look at the excited states in order to see how low in energies these molecular vibrations occur. Another improvment is to add also P-,D-, etc and see if you have other minima (conical intersections). From the knowledge of equilibrium configurations, one can start thinking on how to include rotations (moments of inertia) and what types of bands they generate. One can use the symmetry-adapted wavefunctions as the best basis to optimize the calculation of the whole vibrational spectrum. It is very promising to try to apply the same molecular machinery to complex cluster structure in light nuclei.

\section{References}

[1] L. Fortunato, Journal of Physics: Conf. Series 876012008 (2017)

[2] R. Bijker, F. Iachello, Phys. Rev. Lett. 112, 152501 (2014) 